\title{
MR Image Segmentation based on Local Information Entropy Geodesic Active Contour Model
}

\author{
Jianwei Zhang ${ }^{1}$, Xiang Ma ${ }^{1}$, Yunjie Chen ${ }^{1}$, Lin Fang ${ }^{2}$ and Jin Wang ${ }^{3}$ \\ ${ }^{1}$ School of Math \& Statistics, Nanjing University of Information \\ Science \& Technology, Nanjing 210044, China \\ 2 Intelligent Transportation of Anhui Province Key Lab., Hefei 230088, Ching \\ ${ }^{3}$ School of Computer \& Software, Nanjing University of Information \\ Science \& Technology, Nanjing 210044, China

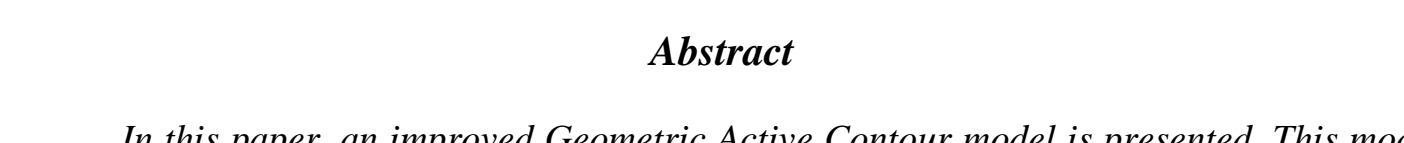

In this paper, an improved Geometric Active Conour model is presented. This model introduced a window function to research the mean information of each pixel's neighbor region, and constructed a novel signed pressureforce function based on the entropy to drive the contour towers the boundary. In order to improve the afficiency and stability of the algorithm, this paper adopts two valued level set method to realize the segmentation process. The experimental results show that this Dethod,can abtain satisfied result even when the images have intensity inhomogenejty@ndweak edges.

Keywords: GAC model, signed pressure foreg function, Local information entropy, Image segmentation

\section{Introduction}

At present, active contour models (ACM) [1] are widely used in image segmentation. This model was initially put forth(by) Kass et al. Generally speaking, the method based on ACM is usually classified into two types: edges-based models [1,2] and region-based methods [3]. The edges-based moders can well segment the target boundary with strong edges, which utilize the gradient information of images, but fail to segment the image with weak boundaries and noise. The region-based models utilize the grey mean or variance can well overcome the iffluence of weak boundaries or noise. However, this models are sensitive to intensity inhomogeneous, thus it can not well segment images with bias field.

Li et at. 4] proposed the local binary fitting (LBF) model, which utilizes the local image infermation to segment objects with intensity inhomogeneity and achieve the better segmentation results. Although the LBF model utilizes local mean information can better overcome the intensity inhomogeneous of images, but it can not well segment the images with strong noise and weak boundaries. Zhang et al. proposed a novel region-based GAC model in paper [5] can well segment the images with noise and weak boundaries. This model constructs a signed pressure force function based on global information to replace the edge-stopping function of the GAC model. This model assumed the grey information of mage is homogeneous, so it can not well segment the images with bias field.

From the research, most medical images local grey information presented gauss distribution and local statistical information can well describe the grey information distribution of the image. This paper can segment the medical images with bias field or weak boundaries and noise better by constructs a novel signed pressure force function based on 
local entropy information which could utilized the local statistical to describe grey information. This method can get better segment result for this model can make full use of the grey information of images.

\section{Background}

Geodesic active contour (GAC) model is put forward by V. Caselles et al. [2] in 1997, and the energy functional of this model is defined as follows:

$$
E^{G A C}=\int_{0}^{L(C)} g(\mid \nabla I[C(s)]) d s
$$

Where $L(C)$ is the length of contour $C, \nabla I$ is the gradient of image $I, \mathrm{~g}(\cdot)$ is the edge stopping function and $s$ is the arc length unit of image. The level set evolution equation corresponding to formula (1) as follows:

$$
\frac{\partial \phi}{\partial t}=g(\mid \nabla I)\left(d i v\left(\frac{\nabla \phi}{|\nabla \phi|}\right)+\alpha\right)+\nabla g \cdot
$$

Where $\operatorname{div}(\cdot)$ is divergence operator, $\alpha$ is a constant. $g(\nabla I)$ is the edge-stopping function that decreasing with the image gradient values 11 increase.

Generally, medical images often exists fuzzy boundaries or weak edges and noise. Because of the GAC model only utilize the gradient informatron but can not utilize comprehensive features of images to drive the evolution curves to segment the target boundary, which lead to the evolution contours over the weak boundaries or fall into the local optimal.

\section{Image Information Entropy}

For the image, $\mathbf{I} \boldsymbol{\Omega} \in \mathbf{R}^{2} \rightarrow \mathbf{R}$ the information of the pixel $x \in \Omega$ is:

$$
\int E(x)=\sum_{i(x-y)} p(I(y)) \log p(I(y))
$$

Where $p(I(y))$ is a Gaussian distribution function in the local region, $w(x-y)$ is a window function which centered at the pixel point $x$. The information entropy can achieve the maximum when the grey distribution is uniform.

Image information entropy [6] also has a widely application in image processing, especiall in image segmentation. Such as the ACM based on regional fitting with local entropy proposed by He Chuanjiang [7] et al. and Amitava Chatterjee et al. utilize maximum fuzzy entropy to segment brain CT images [8].

\section{Signed Pressure Force function based on Local Information Entropy}

Zhang et al. proposed a region-based GAC (RGAC) [5] model, which constructs a signed pressure force $(s p f)$ function replace $\mathrm{g}$ of the GAC model. The definition of $s p f$ as follows:

$$
s p f=\frac{I-\frac{c_{1}+c_{2}}{2}}{\max \left(\mid I-\frac{c_{1}+c_{2}}{2}\right)}
$$


Where $\max (\mid \cdot)$ indicates the absolute maximum of grey difference, grey mean $c_{1}$ and $c_{2}$ respectively defined as follows:

$$
c_{1}=\frac{\iint_{\Omega} I \cdot H(\phi) d x d y}{\iint_{\Omega} H(\phi) d x d y} \quad c_{2}=\frac{\iint_{\Omega} I \cdot(-H(\phi) d x d y}{\iint_{\Omega}(-H(\phi) d x d y}
$$

Because of the RGAC model still rely on intensity homogeneity, and medical image usually include the bias field, so this model cannot to segment the object boundary well.

The grey information of most medical images in local region presents Gaussian distribution according to literature [9]. This paper introduced window function $\mathcal{W}$ to research the grey distribution of local image and introduced the image information entropy into spf to construct a novel drive force function which defined as follows:

$$
\text { lespf }=\frac{\int_{\Omega} w(x-y)\left(E_{m}\left(x+E_{p}, y\right)\right.}{\max \left(\int_{\Omega} w(x-y)\left(E_{m}(x+E, x) d y \mid\right)\right.}
$$

Where $\quad E_{p}(x)=-p_{b}(I(y)) \cdot \log \left(p_{b}(I(y))\right)$ and $E_{m}(x)=-p_{m}(I(y)) \cdot \log \left(p_{m}(I(y))\right)$ respectively present image information of background region and target region, $\Omega$ is the whole image region, $w(x-y)$ is wimaow function $p_{b}(I(y))$ and $p_{m}(I(y))$ respectively present Gaussian function of background and target regions and they respectively computed the posterior probability of background and target regions. According to literature [9], we can know:

$$
p_{b}(I(y))=\frac{\bullet}{2 \pi \sigma_{b}^{2}} \exp \left(-\frac{\left(I(y)-f_{b}\right)}{2 \sigma_{b}^{2}}\right) \quad p_{n}\left((x) \neq \frac{1}{2 \pi \sigma_{m}^{2}} \exp \left(-\frac{\left((y)-f_{m}{ }^{2}\right)}{2 \sigma_{m}^{2}}\right)\right.
$$

Where $f_{b}$ and $f_{m}$ respectively present grey mean of background and target regions:

$$
f_{b}=\frac{\int_{\Omega} w(x-y) I(y) H(\phi(y)) d y}{\int_{\Omega} m(x-y) H(\phi(y)) d y} \quad f_{m}=\frac{\int_{\Omega} w(x-y) y(y)-(H \phi(x(\phi l)}{\left.\int_{\Omega} w(x-y) 1+H \phi(y) d y\right)}
$$

Where $\sigma_{b}$ and $\sigma_{m}$ respectively present variance of background and target regions:

$$
\sigma_{b}^{2}=\frac{\int_{\Omega} w(x-y)\left(I(y)-f_{b}\right)^{2} H(\phi(y)) d y}{\int_{\Omega} w(x-y) H(\phi(y)) d y} \quad \sigma_{m}^{2}=\frac{\int_{\Omega} w(x-y) I\left((y)-f_{m}{ }^{2}\right) 1-t \phi \phi(d y)}{\left.\int_{\Omega} w(x-y) 1+H \phi(y) d y\right)}
$$

In local region, grey changes present Gaussian distribution according literature [9]. Take a point $x$ in contour, suppose $\Omega_{y}$ is a local region which is controlled by window function $w$ and centered at point $x . \Omega_{b}$ and $\Omega_{m}$ respectively present background and object regions that the image region $\Omega_{y}$ divided into inside and external by the contour line. If $x \in \Omega_{b}, E_{p}(x)$ get a smaller value and $E_{m}(x)$ get a bigger value, then lespf $>0$. This situation can drive evolution curves shrinkage to object boundary. In contrast, if $x \in \Omega_{m}$, lespf $<0$, it can drive evolution curves expansion to object boundary. As we can know, the 
grey information in local region satisfies Gaussian distribution, thus the maximum posterior probability of inside and external regions is 1 when the contours approach to object boundary. Moreover, we can know that $-p_{b}(I(y)) \log p_{b}(I(y)) \approx 0$ and $-p_{m}(I(y)) \log p_{m}(I(y)) \approx 0$, therefore lespf $\approx 0$ and the contour line will stop evolution at the object boundary. From the formula (6), the range of variation of function lespf is $[-1,1]$ satisfies definition of spf function based on the above discussion.

Take lespf instead of spf in the RGAC model; get the corresponding gradient descent flow equations as follows:

$$
\frac{\partial \phi}{\partial t}=\operatorname{lespf} \cdot|\nabla \phi| \cdot\left[\operatorname{div}\left(\frac{\nabla \phi}{|\nabla \phi|}\right)+\alpha\right]+\nabla \operatorname{lespf} \cdot \nabla \phi
$$

Because of grey values in local region of medical images chlanges slowly, the derivative of lespf approach to 0 , writing as Vlespf $\approx 0$. Simultaneousty, $\operatorname{div}\left(\frac{\nabla \phi}{|\nabla \phi|}\right) \cdot|\nabla \phi|=\Delta \phi$, and $G_{\sigma} * \phi$ can be used to approach $\Delta \phi$ in this paper. This paper hrough utilize Gauss filter instead of curvature regularization. From the above discussion, formula, (10) ean be written brief as:

\section{Experimental Results and Analysis}

Figure 1 is the segmentation of left ventricle MR image. Figure 1(a)-(b) is the original image with initial curve and the true segmentation result. Figure 1(c) is the segmentation result of $\mathrm{C}-\mathrm{V}$ model. This model can not well segment the images with bias field or weak boundaries. Figure $1(d)$ is the result df GAC model which iterate 600 times. This model can only precede single direction evolution. Figure 1(e) is the result of RGAC model which iterate 100 times. This modetcan easy occur over-segmentation. Figure 1(f)-(g) respectively presents the result of LBFand LGD model which iterate 100 and 200 times. The parameter of them is $\lambda_{1}=1.0, \lambda_{2}=1.0$ and kernel parameter sigma $=15$. Both of them can well segment images with bias fied, but fail to segment noise image. Figure 1(h) is segmentation result of this paper method iterate 100 times with parameter sigma $=15$ and $\alpha=100$. This method can well segment the images with bias field and weak boundaries.

In order to better respond or compare segmentation accuracy and segmentation efficiency of each nodel, we utilize 20 groups left ventricle MR images for experiment segmentation test. The size of image is $110 \times 110$, and utilize the Jaccard Similarity (JS) index [10] to quantitative analysis the segmentation accuracy.

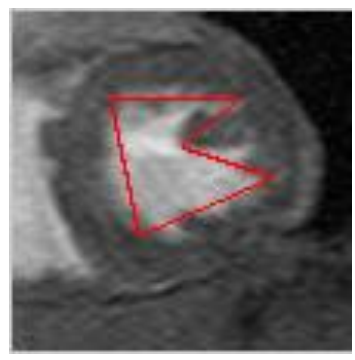

(a)

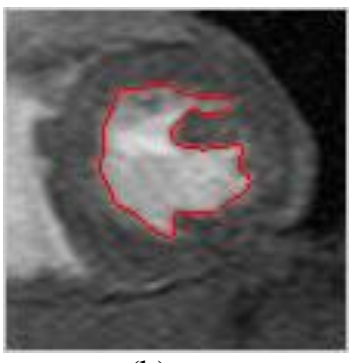

(b)

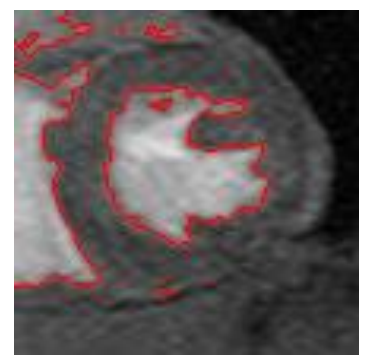

(c)

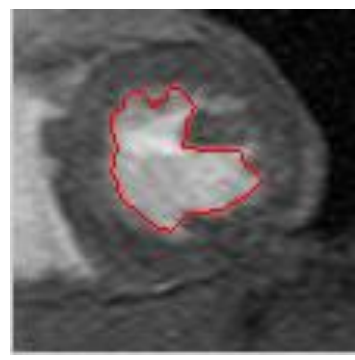

(d) 


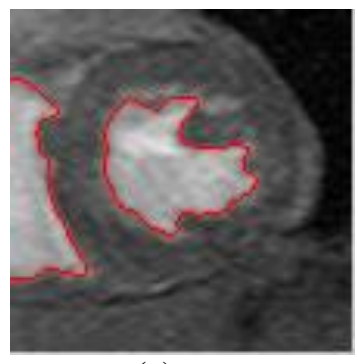

(e)

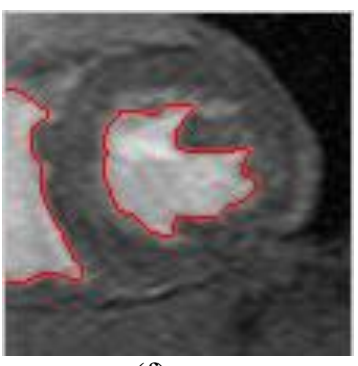

(f)

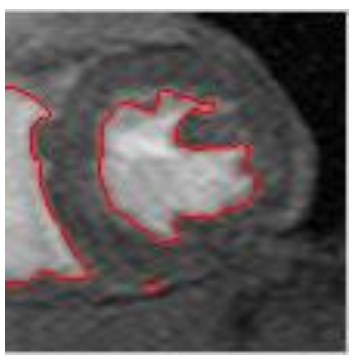

(g)

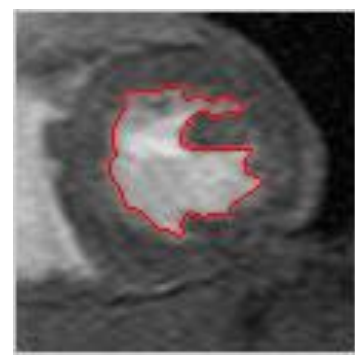

(h)

Figure 1. The segmentation result of left ventricle magnetic resonance (MR) image. Figure 1(a)-(b) respectively present original image with initial curve and accurate segmentation result. Figure $1(d)-(h)$ respectively presentsegmentation result of C-V model, GAC model, RGAC model, LBF model, LGD model and this paper method

As shown in the figure, the segmentation accuracy of this paper method is more than $90 \%$. The $\mathrm{C}-\mathrm{V}$ model can not well segment the images with bias field. The GAC model can easily produces boundary leakage. The RGAC model fails to segment the images with bias field. Due to lots of convolution operation, the segmentation efficiency of LBF and LGD model is lower. This paper method can well segment the images with bias field. Due to this method adopt window function to computer, the average iteration time no more than 3 seconds and iteration times less than 200 times This situation is stily in reasonable range.

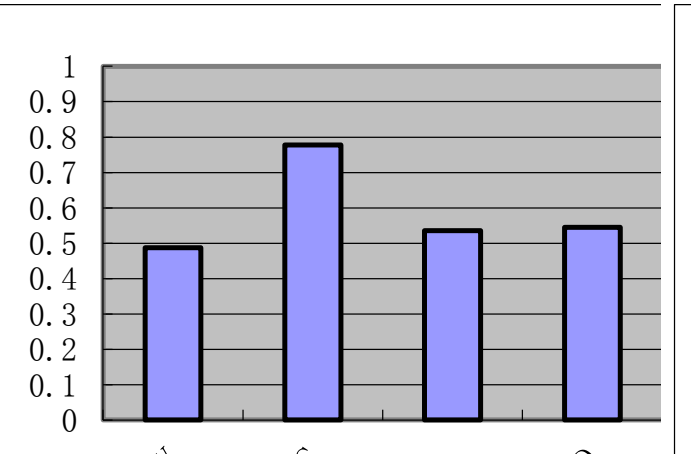

Figure 2. Segmentation Accuracy

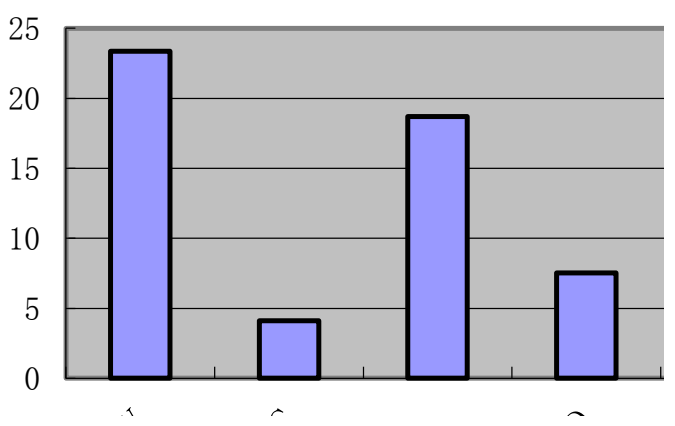

Figure 3. Segmentation Efficiency

Figure 4 is the segmentation result of left ventricle MRI with sigma for [3, 9, 15, 21]. Initral Image exits of noise, bias field, weak boundaries and labeled line. Figure 4(1)-(2) respectively present the origin image with initial curve and the accurate segmentation result. Figure 4(3) is the result of RGAC model iterates 100 times with the different sigma. This model can well overcome noise and weak boundaries, but easy cause to segment homogenous images wrong. Figure 4(4)-(5) respectively present the result of LBF and LGD model iterates 100 and 200 times with the parameters $\lambda_{1}=1.0, \lambda_{2}=1.0$. The LBF model and LGD model can not well segment the images included bias field with the sigma increase. Figure 4(6) is the result of this paper method iterates 150 times with parameter $\alpha=3$. This method can well segment the images with the intensity inhomogeneous. Figure 5 is the segmentation result of this paper method iterates 150 times with sigma $=15$ and $\alpha=[1.5,3,4.5,6]$. 
Figure 6 and Figure 7 respectively present the segmentation accuracy and efficiency with the sigma. From the figure, this paper method is obviously better than RGAC, LBF and LGD model. With the increase of sigma, the segmentation accuracy of LBF and LGD model decrease.

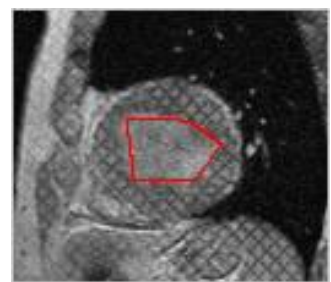

(1) Orign Image with Initial Curves

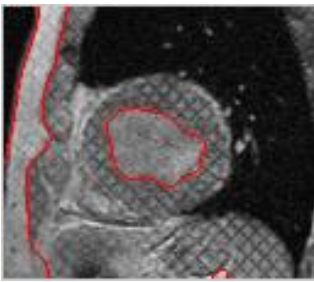

(a.1) $\operatorname{sigma}=3$

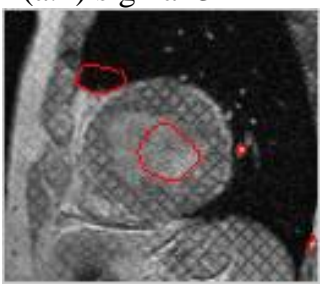

(b.1) sigma=3

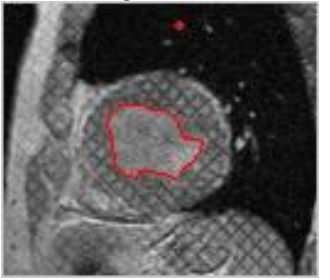

(c.1) sigma=3

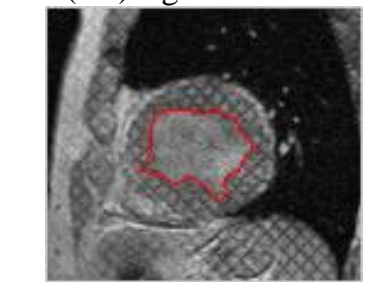

(d.1) sigma=3

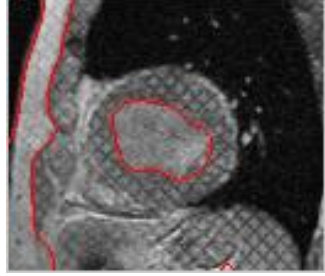

(a.2) $\operatorname{sigma}=9$

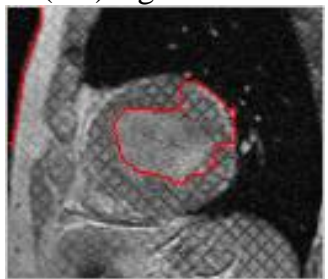

(b.2) sigma=9

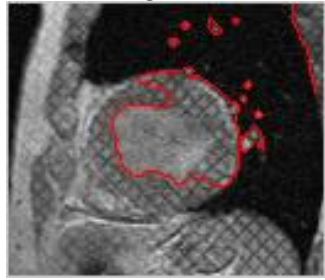

(c. 2$)$ sigma $=9$

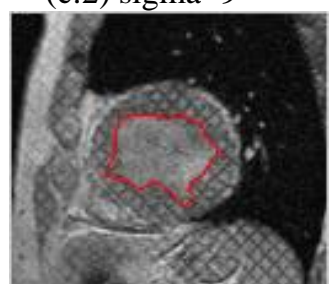

(d.2) sigma $=9$
(2)

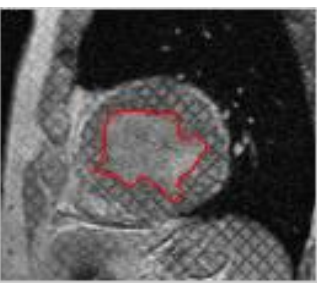

Accurate Segmentation Resalt

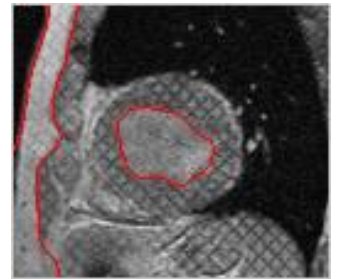

(a.3) sigma $=15$

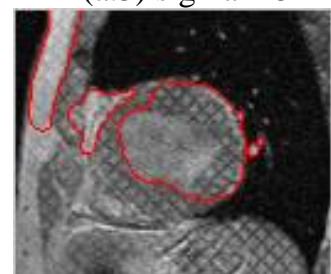

(b.3) sigma $=15$

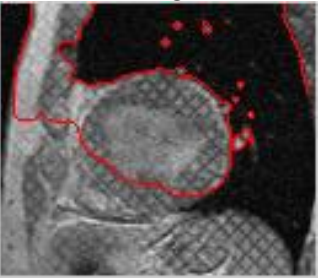

(c.3) $\operatorname{sigma}=15$

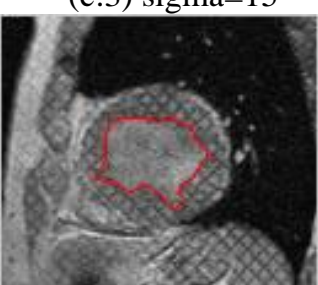

(d.3) sigma $=15$

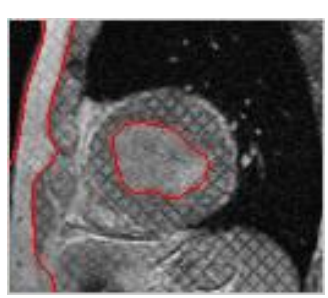

(a.4) $\operatorname{sigma}=21$

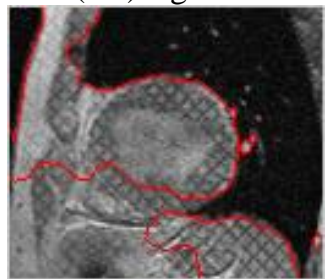

(b.4) sigma $=21$

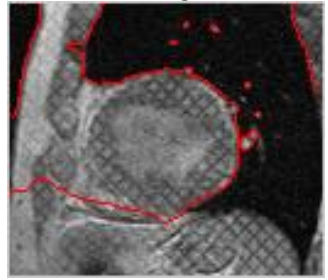

(c.4) sigma=21

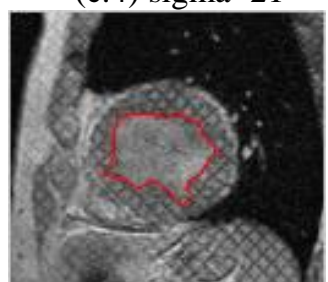

(d.4) $\operatorname{sigma}=21$

Figure 4. The first row is the segmentation result of origin image with initial curves. The second to fifth row respectively present the segmentation result of RGAC model, LBF model, LGD model and this method based on the size of sigma is $[3,9,15,21]$ 


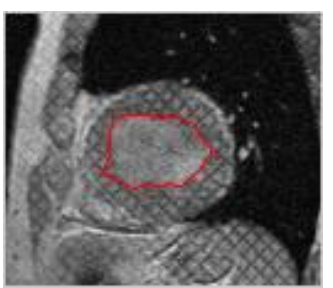

(a) alpha $=1.5$

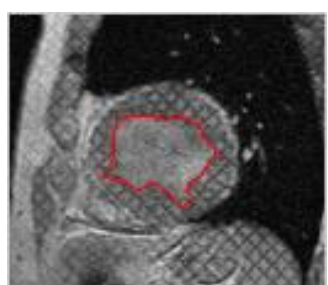

(b) alpha $=3$

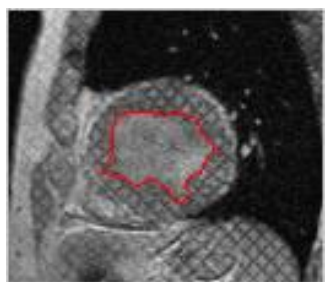

(c) alpha $=4.5$

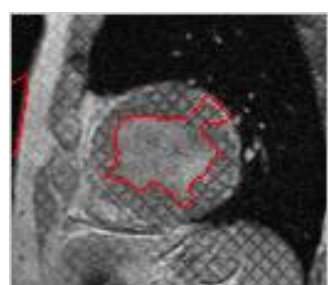

(d) alpha $=6$

Figure 5. The segmentation result with the influence of the parameter $\alpha$ in this paper

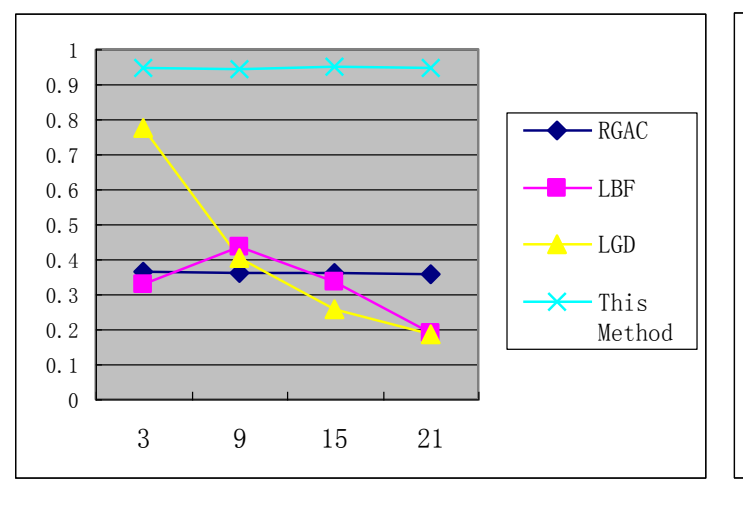

Figure 6. The influence of segmentation accutare with sigma

\section{Conclusions}

This paper proposed an improved GAC model for the images with noise, bias field and weak boundaries. This pape model utilizes the window function to investigate the grey information of local region, while introduce the local information entropy to describe the distribution of the grey information. Compared with GAC model, this method can achieve the high segmentation accuracy. Compared with C-V and RGAC model, this method can well segment the images with bias field. And compared with LBF and LGD model, this method has more choice of kernel parameters.

\section{Acknowledgements}

This research work was supported by the National Nature Science Foundation of China (61003209, 61173072), and the Natural Science Foundation of Jiangsu Province (BK2011824, BK2012461).

\section{References}

[1] M. Kass, A. Witkin and D. Terzopulos, "Snake active contours models", International Journal of Computer Vision, vol. 1, no. 3, (1988).

[2] V. Caselles, R. Kimmel and G. Sapiro, "Geodesic active contours", International Journal of Computer Vision, vol. 22, no. 1, (1997).

[3] T. Chan and L. Vese, "Active contours without edges", IEEE Transactions on Image processing, vol. 10, no. 2, (2001). 
[4] C. Li, C .-Y. Kao, et al., "Implicit Active Contours Driven by Local Binary Fitting Energy", Computer Vision and Pattern Recognition, (2007) June 17-22, Minneapolis, MN, USA.

[5] K. Zhang, L. Zhang, et al., "Active contours with selective local or global segmentation: A new formulation and level set method", Image and Vision computing, vol. 28, (2010), pp. 668-676.

[6] C. E. Shannon, "A Mathematical Theory of Communication”, The Bell System Technical Journal, vol. 27, (1948).

[7] C. He, Y. Wang and Q. Chen, "Active contours driven by weighted region-scalable fitting energy based on local entropy", Signal Processing, vol. 92, no. 2, (2012).

[8] A. Chatterjee, P. Siarry, A. Nakib and R. Blanc, "An improved biogeography based optimization approach for segmentation of human head CT-scan images employing fuzzy entropy”, Engineering Applications of Artificial Intelligence, vol. 25, no. 8, (2012).

[9] L. Wang, L. He, A. Mishra and C. Li, "Active contours driven by local Gaussian distribution fitting energy", Signal Processing, vol. 89, no. 12, (2009).

[10] U. Vork, F. Pernnus and B. Likar, "A review of methods for correction of intensity inhomogeneity in MRI", IEEE Transactions on Medical Imaging, vol. 26, no. 3, (2007).

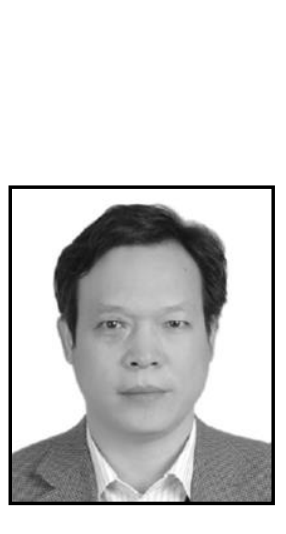

\section{Jianwei Zhang}

$\mathrm{He}$ is Professor at the College of Mathematics and Physics, Nanjing University of Information Science and Technology. His research interest covers pattern recognition, artificyal intelligence, and remote sensing information processing.

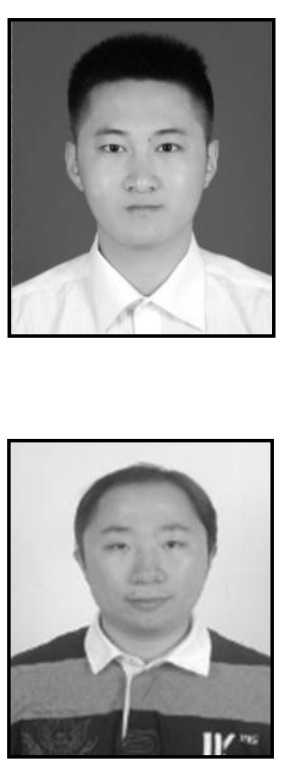

Xiang Ma

Authors

Graduate students, Nanjing University of Information Science and Technology. His research interest covers image processing, pattern recognition
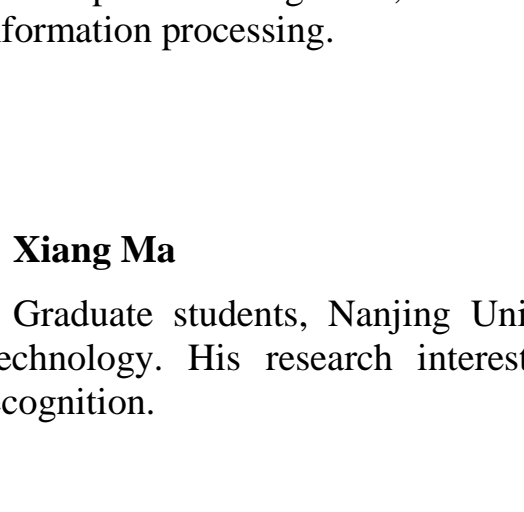

\section{YunJie Chen}

He received the B.S. and M.S. degree in the Applied Mathematics from Nanjing University of Information Science and Technology, China in 2002 and 2005, respectively. He received Ph.D. degree in Pattern Recognition and Intelligent Systems from NUST. Now, he is an associate professor in NUIST. His research interests include image processing, pattern recognition and numerical analysis. 


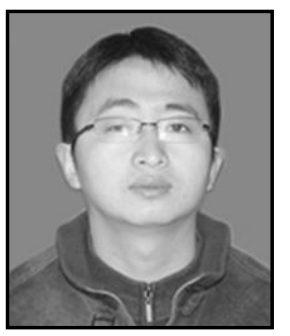

\section{Lin Fang}

He received M.S. degree in Applied Mathematics from Nanjing University of Information Science and Technology. Now, he works in Intelligent Transportation of Anhui Province Key Lab. His research interests include image processing, pattern recognition and numerical analysis.

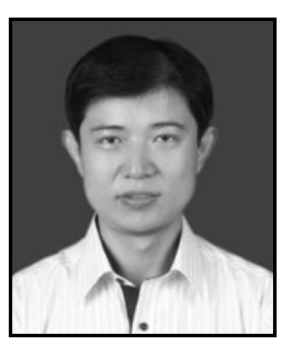

\section{Jin Wang}

He received the B.S. and M.S. degree in the Electronical Engineering from Nanjing University of Posts and Telecommunications, China in 2002 and 2005, respectively. He recejved Ph.D. degree in the Ubiquitous Computing laboratory from the Computen Engineering Department of Kyung Hee University Korea in 2010. Now, he is a professor in the Computer and Software Institute, Nanjing University of Information Science and technology. His research interests mainly include routing method and algorithm design, performance evaluation and optimization for wireless ad hoc and sensor networks He is a member of the IEEE and ACM.

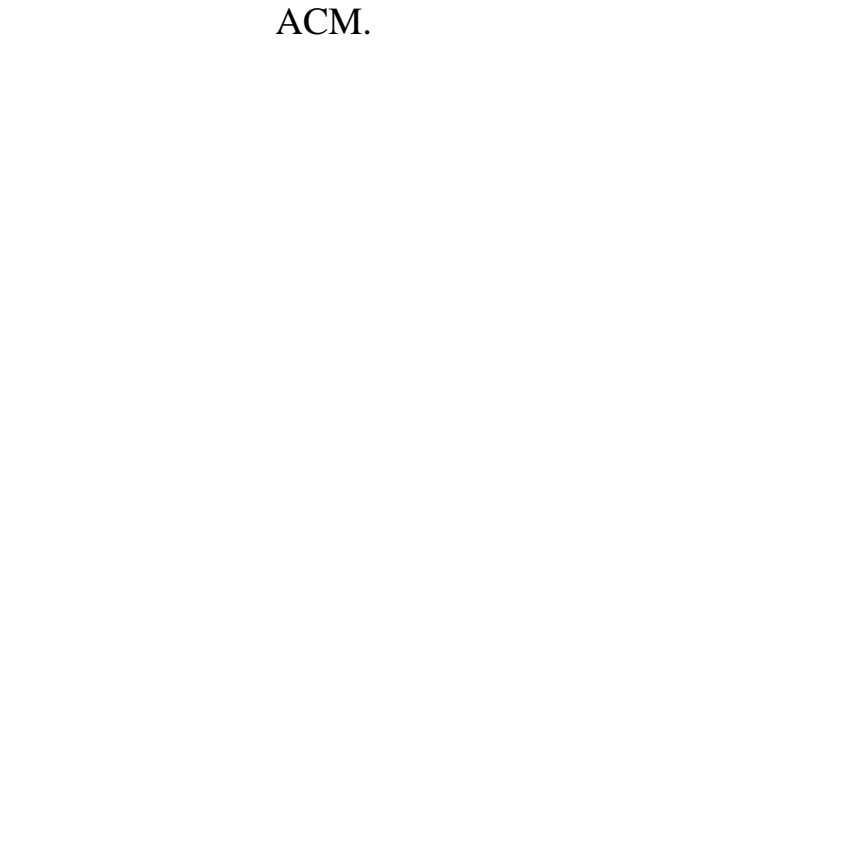


International Journal of Multimedia and Ubiquitous Engineering Vol.9, No.4 (2014)

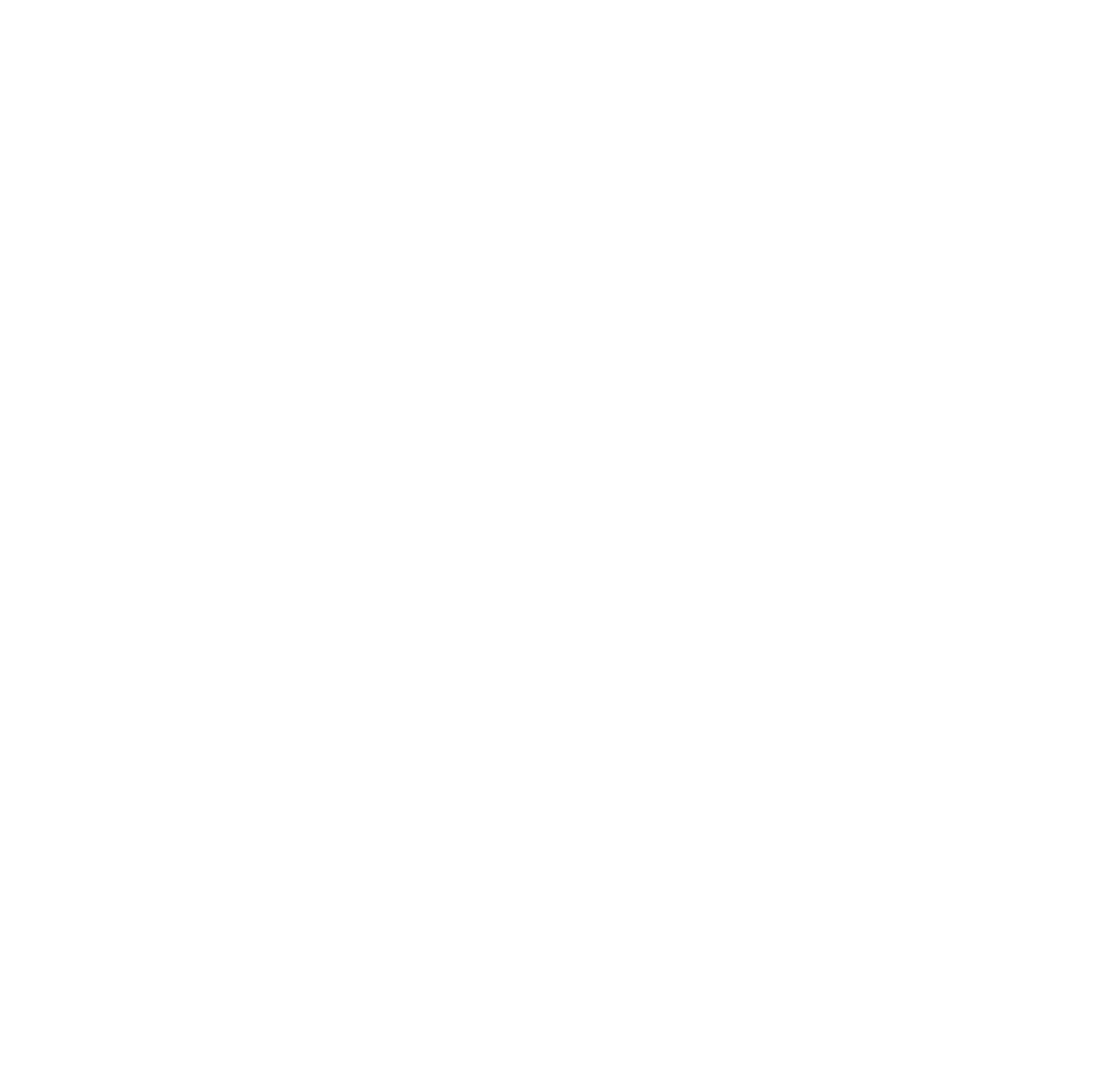

